\title{
A Characterization of a Local Vector Valued Bollobás Theorem
}

\author{
Sheldon Dantas@ and Abraham Rueda Zoca@
}

\begin{abstract}
In this paper, we are interested in giving two characterizations for the so-called property $\mathbf{L}_{o, o}$, a local vector valued Bollobás type theorem. We say that $(X, Y)$ has this property whenever given $\varepsilon>0$ and an operador $T: X \rightarrow Y$, there is $\eta=\eta(\varepsilon, T)$ such that if $x$ satisfies $\|T(x)\|>1-\eta$, then there exists $x_{0} \in S_{X}$ such that $x_{0} \approx x$ and $T$ itself attains its norm at $x_{0}$. This can be seen as a strong (although local) Bollobás theorem for operators. We prove that the pair $(X, Y)$ has the $\mathbf{L}_{o, o}$ for compact operators if and only if so does $(X, \mathbb{K})$ for linear functionals. This generalizes at once some results due to D. Sain and J. Talponen. Moreover, we present a complete characterization for when $\left(X \widehat{\otimes}_{\pi} Y, \mathbb{K}\right)$ satisfies the $\mathbf{L}_{o, o}$ for linear functionals under strict convexity or Kadec-Klee property assumptions in one of the spaces. As a consequence, we generalize some results in the literature related to the strongly subdifferentiability of the projective tensor product and show that $\left(L_{p}(\mu) \times L_{q}(\nu) ; \mathbb{K}\right)$ cannot satisfy the $\mathbf{L}_{o, o}$ for bilinear forms.
\end{abstract}

Mathematics Subject Classification. Primary 46B04; Secondary 46A32, 46B20, 46B10.

Keywords. Norm attaining operators, Bishop-Phelps theorem, BishopPhelps-Bollobás theorem, projective tensor products, compact operators.

\section{Introduction}

It has now been 60 years since Bishop and Phelps proved that every bounded linear functional can be approximated by norm-attaining ones [2]. Since then, several researchers have been working in norm-attaining theory in many different directions and it is out of doubt one of the most traditional topics in Functional Analysis nowadays. Bollobás [3] pushed further the Bishop-Phelps 
theorem by proving that if $\varepsilon>0$, then there exists $\eta(\varepsilon)>0$ such that whenever $x^{*} \in S_{X^{*}}$ and $x \in S_{X}$ satisfy $\left|x^{*}(x)\right|>1-\eta(\varepsilon)$, then there exist a new functional $x_{0}^{*} \in S_{X^{*}}$ and a new element $x_{0} \in S_{X}$ such that

$$
\left|x_{0}^{*}\left(x_{0}\right)\right|=1, \quad\left\|x_{0}-x\right\|<\varepsilon, \quad \text { and } \quad\left\|x_{0}^{*}-x^{*}\right\|<\varepsilon
$$

(our notation is standard and can be found in Sect. 1.1 at the end of this introduction). Let us notice that the Bishop-Phelps theorem plays an important role in non-reflexive spaces since otherwise every functional attains its norm. On the other hand, Bollobás theorem does make sense in the reflexive setting and, in this case, the functional $x^{*}$ necessarily attains its norm; so it would be natural to wonder whether a version of Bollobás theorem without changing the initial functional $x^{*}$ holds in general, that is, whether it is possible to take $x_{0}^{*}=x^{*}$ in (1.1). In a more general situation, we are wondering the following: given $\varepsilon>0$, is it possible to find $\eta(\varepsilon)>0$ such that whenever $T \in \mathcal{L}(X, Y)$ with $\|T\|=1$ and $x \in S_{X}$ satisfy $\|T(x)\|>1-\eta(\varepsilon)$, one can find a new element $x_{0} \in S_{X}$ such that $\left\|T\left(x_{0}\right)\right\|=1$ and $\left\|x_{0}-x\right\|<\varepsilon$ ? It is easy to see that the pair $(X, \mathbb{K})$ satisfies it whenever $X$ is a uniformly convex Banach space and it turns out that this is in fact a characterization for uniformly convex spaces (see [13, Theorem 2.1]). Nevertheless, there is no way of getting such a similar statement for linear operators: indeed, the authors in [7] proved that if $X$ and $Y$ are real Banach spaces of dimension greater than or equal to 2 , then the pair $(X, Y)$ always fails such a property. Therefore, the only hope for getting positive results in the context of operators would be by considering a weakening of the mentioned property and that was done in $[4,8,9,16,17]$ (and more recently in $[5,6]$ as a tool to get positive results on different norm-attainment notions). More specifically, we have the following property.

Definition 1.1. Let $X, Y$ be Banach spaces. We say that the pair $(X, Y)$ has the $\mathbf{L}_{o, o}$ for operators if given $\varepsilon>0$ and $T \in \mathcal{L}(X, Y)$ with $\|T\|=1$, there exists $\eta(\varepsilon, T)>0$ such that whenever $x \in S_{X}$ satisfies $\|T(x)\|>1-\eta(\varepsilon, T)$, there exists $x_{0} \in S_{X}$ such that

$$
\left\|T\left(x_{0}\right)\right\|=1 \quad \text { and } \quad\left\|x_{0}-x\right\|<\varepsilon .
$$

Notice that if the pair $(X, Y)$ satisfies such a property, then, in particular, every operator has to be norm-attaining. Consequently, the Banach space $X$ must be reflexive by James' theorem. By using a result due to Godefroy et al. [12] and a characterization by Franchetti and Payá [11], it turns out that the pair $(X, \mathbb{K})$ has the $\mathbf{L}_{o, o}$ for linear functionals if and only if $X^{*}$ is strongly subdifferentiable (SSD, for short; see its definition below). On the other hand, at the same way that it happens in the classical norm-attaining theory (see, for instance, [14]), the $\mathbf{L}_{o, o}$ was studied for compact operators [16,17]: we say that $(X, Y)$ has the $\mathbf{L}_{o, o}$ for compact operators if given $\varepsilon>0$ and a normone compact operator $T$, there is $\eta(\varepsilon, T)>0$ such that whenever $x \in S_{X}$ satisfies $\|T(x)\|>1-\eta(\varepsilon, T)$, there exists $x_{0} \in S_{X}$ satisfying conditions (1.2). It is known that whenever $X$ is strictly convex and $Y$ is an arbitrary Banach 
space, the pair $(X, Y)$ has the $\mathbf{L}_{o, o}$ for compact operators if and only if the dual $X^{*}$ is Fréchet differentiable (see [17, Theorem 2.3]); and when $X$ satisfies the Kadec-Klee property then $(X, Y)$ has the $\mathbf{L}_{o, o}$ for compact operators for every Banach space $Y$ (see [16, Theorem 2.12]).

Our first aim in the present paper is to generalize [16, Theorem 2.12] and [17, Theorem 2.3] at once. Indeed, we have the following theorem.

Theorem A. Let $X$ be a reflexive Banach space. The following are equivalent.

(i) The pair $(X, Y)$ satisfies the $\mathbf{L}_{o, o}$ for compact operators for every Banach space $Y$.

(ii) The pair $(X, \mathbb{K})$ has the $\mathbf{L}_{o, o}$ (equivalently, $X^{*}$ is $\left.S S D\right)$.

Our second main result deals with a strengthening of the $\mathbf{L}_{o, o}$ in the context of bilinear forms (see [8]).

Definition 1.2. [8, Definition 2.1]. Let $X, Y$ be Banach spaces. We say that $(X \times Y ; \mathbb{K})$ has the $\mathbf{L}_{o, o}$ for bilinear forms if given $\varepsilon>0$ and $B \in \mathcal{B}(X \times Y ; \mathbb{K})$ with $\|B\|=1$, there exists $\eta(\varepsilon, B)>0$ such that whenever $(x, y) \in S_{X} \times S_{Y}$ satisfies $|B(x, y)|>1-\eta(\varepsilon, B)$, there exists $\left(x_{0}, y_{0}\right) \in S_{X} \times S_{Y}$ such that

$$
\left|B\left(x_{0}, y_{0}\right)\right|=1, \quad\left\|x_{0}-x\right\|<\varepsilon, \quad \text { and } \quad\left\|y_{0}-y\right\|<\varepsilon .
$$

It is known that $(X \times Y ; \mathbb{K})$ satisfies the $\mathbf{L}_{o, o}$ for bilinear forms whenever

(a) $X, Y$ are finite dimensional;

(b) $X$ is finite dimensional and $Y$ is uniformly convex;

(c) $X=\ell_{p}$ and $Y=\ell_{q}$ if and only if $p>q^{\prime}$, where $q^{\prime}$ is the conjugate index of $q$.

(see Proposition 2.2.(a), Lemma 2.6, and Theorem 2.7.(b) of [8], respectively). By observing items (a), (b), and (c) above, one might think that the reflexivity of the projective tensor product $X \widehat{\otimes}_{\pi} Y$ plays an important role here (notice that (c) gives the result for $\ell_{p}$-spaces exactly when the projective tensor product $\ell_{p} \widehat{\otimes}_{\pi} \ell_{q}$ is reflexive (see [15, Corollary 4.24])). And this is indeed not a coincidence: we have the following result, which gives a complete characterization for the $\mathbf{L}_{o, o}$ in terms of the reflexivity of $X \widehat{\otimes}_{\pi} Y$ and also relates the $\mathbf{L}_{o, o}$ in different classes of functions under strict convexity or Kadec-Klee property assumptions on $X$. For the necessary terminology on approximation properties, we send the reader to the very end of Sect. 1.1.

Theorem B. Let $X$ be a strictly convex Banach space or a Banach space satisfying the Kadec-Klee property. Let $Y$ be an arbitrary Banach space. Assume that either $X$ or $Y$ enjoys the $A P$, or that the pair $\left(X, Y^{*}\right)$ satisfies the pointwise-BCAP. The following are equivalent.

(a) $\mathcal{L}\left(X, Y^{*}\right)=\mathcal{K}\left(X, Y^{*}\right)$ and both $(X, \mathbb{K}),(Y, \mathbb{K})$ have the $\mathbf{L}_{o, o}$ for linear functionals.

(b) $X \widehat{\otimes}_{\pi} Y$ is reflexive and both $(X, \mathbb{K}),(Y, \mathbb{K})$ have the $\mathbf{L}_{o, o}$ for linear functionals. 
(c) $(X \times Y ; \mathbb{K})$ has the $\mathbf{L}_{o, o}$ for bilinear forms.

(d) $\left(X \widehat{\otimes}_{\pi} Y, \mathbb{K}\right)$ has the $\mathbf{L}_{o, o}$ for linear functionals.

As a consequence of Theorem $\mathrm{B}$, we have that $\left(L_{p}(\mu) \times L_{q}(\nu) ; \mathbb{K}\right)$ cannot satisfy the $\mathbf{L}_{o, o}$ for bilinear forms for every $1<p, q<\infty$ and for not purely atomic measures $\mu, \nu$, since $L_{p}(\mu) \widehat{\otimes}_{\pi} L_{q}(\nu)$ is never reflexive (see [15, Theorem 4.21 and Corollary 4.22]). We conclude the paper with a discussion about the relation between the $\mathbf{L}_{o, o}$ in $\mathcal{B}(X \times Y, \mathbb{K})$ when we view $\mathcal{B}(X \times Y, \mathbb{K})$ as an space of operators or a dual space.

\subsection{Terminology and Background}

Here will be working with Banach spaces over the real or complex field $\mathbb{K}$. The unit ball and unit sphere of a Banach space $X$ are denoted by $B_{X}$ and $S_{X}$, respectively. The symbols $\mathcal{L}(X, Y)$ and $\mathcal{B}(X \times Y ; \mathbb{K})$ stand for the (bounded) linear operators and bilinear forms, respectively. When $Y=\mathbb{K}$, $\mathcal{L}(X, Y)$ becomes simply $X^{*}$, the topological dual space of $X$. We say that $T \in \mathcal{L}(X, Y)$ attains its norm if $\left\|T\left(x_{0}\right)\right\|=\|T\|$ for some $x_{0} \in S_{X}$ and we say that $B \in \mathcal{B}(X \times Y ; \mathbb{K})$ attains its norm if $\left|B\left(x_{0}, y_{0}\right)\right|=\|B\|$ for some $\left(x_{0}, y_{0}\right) \in S_{X} \times S_{Y}$.

The norm of $X$ is said to be strongly subdifferentiable (SSD, for short) at the point $x \in X$ if the one-side limit

$$
\lim _{t \rightarrow 0^{+}} \frac{\|x+t h\|-\|x\|}{t}
$$

exists uniformly for $h \in B_{X}$. Let us notice that the norm of $X$ is Fréchet differentiable at $x$ if and only if it is Gâteux differentiable and SSD at $x$. When we say that $X$ is SSD we mean that the norm of $X$ is SSD at every $x \in S_{X}$.

The projective tensor product of two Banach spaces $X$ and $Y$ is the completion of $X \otimes Y$ endowed with the norm given by

$$
\|z\|_{\pi}=\inf \left\{\sum_{n=1}^{\infty}\left\|x_{n}\right\|\left\|y_{n}\right\|: \sum_{n=1}^{\infty}\left\|x_{n}\right\|\left\|y_{n}\right\|<\infty, z=\sum_{n=1}^{\infty} x_{n} \otimes y_{n}\right\} .
$$

We denote the projective tensor product of $X$ and $Y$ endowed with the above norm by $X \widehat{\otimes}_{\pi} Y$. It is well-known (and we will be using these facts with no explicit mention throughout the paper) that $\|x \otimes y\|=\|x\|\|y\|$ for every $x \in X$ and $y \in Y$, and that the closed unit ball of $X \widehat{\otimes}_{\pi} Y$ is the closed convex hull of $B_{X} \otimes B_{Y}=\left\{x \otimes y: x \in B_{X}, y \in B_{Y}\right\}$. Moreover, we have that $\left(X \widehat{\otimes}_{\pi} Y\right)^{*}=$ $\mathcal{B}(X \times Y ; \mathbb{K})$ under the action of a bounded bilinear form $B$ as a bounded linear functional on $X \widehat{\otimes}_{\pi} Y$ given by

$$
\left\langle B, \sum_{n=1}^{\infty} x_{n} \otimes y_{n}\right\rangle=\sum_{n=1}^{\infty} B\left(x_{n}, y_{n}\right)
$$


and $\left(X \widehat{\otimes}_{\pi} Y\right)^{*}=\mathcal{L}\left(X, Y^{*}\right)$ under the action of a bounded linear operator $T$ as a bounded linear functional on $X \widehat{\otimes}_{\pi} Y$ given by

$$
\left\langle T, \sum_{n=1}^{\infty} x_{n} \otimes y_{n}\right\rangle=\sum_{n=1}^{\infty}\left\langle y_{n}, T\left(x_{n}\right)\right\rangle .
$$

Analogously, we have that $\left(X \widehat{\otimes}_{\pi} Y\right)^{*}=\mathcal{L}\left(Y, X^{*}\right)$.

Recall that a Banach space is said to have the approximation property (AP, in short) if for every compact subset $K$ of $X$ and every $\varepsilon>0$, there exists a finite-rank operator $T: X \longrightarrow X$ such that $\|T(x)-x\| \leq \varepsilon$ for every $x \in K$. We also make use of the so-called pointwise bounded compact approximation property (pointwise-BCAP, for short) defined recently in [10]: we say that a pair of Banach space $(X, Y)$ has the pointwise-BCAP if for every operator $T \in \mathcal{L}(X, Y)$, there exists a constant $\lambda_{T} \geq 1$ such that $T \in \lambda_{T} \overline{B_{\mathcal{K}(X, Y)}} \tau_{c}$, where $\tau_{c}$ denotes the topology of compact convergence in $\mathcal{L}(X, Y)$. We refer the reader to [15] for background on the beautiful tensor products of Banach spaces and approximation properties theories.

Finally, let us recall that a Banach space $X$ satisfies the Kadec-Klee property if the weak and the norm topologies coincide in the unit sphere of $X$.

\section{Proofs of Theorems A and B}

We start this section by giving the proof of Theorem A.

Proof of Theorem A. (i) $\Rightarrow$ (ii). Suppose that $(X, Y)$ has the $\mathbf{L}_{o, o}$ for compact operators. Let $\varepsilon>0$ and $x^{*} \in S_{X^{*}}$ be given, and let us prove that $(X, \mathbb{K})$ has the $\mathbf{L}_{o, o}$ for linear functionals. Define $T: X \longrightarrow Y$ by $T(x):=x^{*}(x) y_{0}$ for some $y_{0} \in S_{Y}$. Then, $\|T\|=\left\|x^{*}\right\|=1$ and $T$ is compact. By hypothesis, there is $\eta(\varepsilon, T)>0$ witnessing the definition of the property $\mathbf{L}_{o, o}$. Let us set $\eta\left(\varepsilon, x^{*}\right):=\eta(\varepsilon, T)>0$. Let $x_{0} \in S_{X}$ be such that $\left|x^{*}\left(x_{0}\right)\right|>1-\eta(\varepsilon, T)$. Then, $\left\|T\left(x_{0}\right)\right\|=\left\|x^{*}\left(x_{0}\right) y_{0}\right\|=\left|x^{*}\left(x_{0}\right)\right|>1-\eta(\varepsilon, T)$ and by the assumption there is $x_{1} \in S_{X}$ such that $\left\|T\left(x_{1}\right)\right\|=1$ and $\left\|x_{1}-x_{0}\right\|<\varepsilon$. Then, $\left|x^{*}\left(x_{1}\right)\right|=\left\|T\left(x_{1}\right)\right\|=$ 1 and $\left\|x_{1}-x_{0}\right\|<\varepsilon$, that is, $(X, \mathbb{K})$ has the $\mathbf{L}_{o, o}$ for linear functionals.

(ii) $\Rightarrow$ (i). Suppose that $(X, \mathbb{K})$ has the $\mathbf{L}_{o, o}$ for linear functionals. By contradiction, suppose that there exist $\varepsilon_{0}>0, T \in \mathcal{K}(X, Y)$ with $\|T\|=1$, and $\left(x_{n}\right) \subseteq S_{X}$ such that

$$
1 \geq\left\|T\left(x_{n}\right)\right\| \geq 1-\frac{1}{n}
$$

but satisfying that dist $\left(x_{n}, \mathrm{NA}(T)\right) \geq \varepsilon_{0}$, where $\mathrm{NA}(T)=\left\{x \in S_{X}:\|T(x)\|=\right.$ $\|T\|\}$. Since $X$ is reflexive and $\left(x_{n}\right)_{n=1}^{\infty}$ is bounded, we may (and we do) assume that $x_{n} \stackrel{w}{\longrightarrow} x_{0}$ for some $x_{0} \in B_{X}$. Since $T$ is a compact operator, we have that $T\left(x_{n}\right) \stackrel{\|\cdot\|}{\longrightarrow} T\left(x_{0}\right)$. By $(2.1)$, we have that $\left\|T\left(x_{0}\right)\right\|=1$ and, in particular, $x_{0} \in S_{X}$. Let us take $y_{0}^{*} \in S_{Y^{*}}$ to be such that $y^{*}\left(T\left(x_{0}\right)\right)=\left\|T\left(x_{0}\right)\right\|=1$. Consider $x_{0}^{*}:=T^{*} y_{0}^{*} \in S_{X^{*}}$. Then $x_{0}^{*}\left(x_{0}\right)=T^{*} y_{0}^{*}\left(x_{0}\right)=y^{*}\left(T\left(x_{0}\right)\right)=1$. Since 
$x_{n} \stackrel{w}{\longrightarrow} x_{0}$, we have that $x_{0}^{*}\left(x_{n}\right) \longrightarrow x_{0}^{*}\left(x_{0}\right)=1$ as $n \rightarrow \infty$. Since $(X, \mathbb{K})$ has the $\mathbf{L}_{o, o}$ for linear functionals, there is $\left(x_{n}^{\prime}\right) \subseteq S_{X}$ such that $x_{0}^{*}\left(x_{n}^{\prime}\right)=1$ for every $n \in \mathbb{N}$ and $\left\|x_{n}^{\prime}-x_{n}\right\| \rightarrow 0$. This shows that

$$
1=x_{0}^{*}\left(x_{n}^{\prime}\right)=T^{*} y_{0}^{*}\left(x_{n}^{\prime}\right)=y_{0}^{*}\left(T\left(x_{n}^{\prime}\right)\right),
$$

that is, $1=y_{0}^{*}\left(T\left(x_{n}^{\prime}\right)\right) \leq\left\|T\left(x_{n}^{\prime}\right)\right\| \leq\|T\|=1$, so, $\left\|T\left(x_{n}^{\prime}\right)\right\|=1$ and then $x_{n}^{\prime} \in \mathrm{NA}(B)$. The convergence $\left\|x_{n}^{\prime}-x_{n}\right\| \rightarrow 0$ yields the desired contradiction.

Remark 2.1. [17, Theorem 2.3] says that $X$ is strictly convex and the pair $(X, Y)$ has the $\mathbf{L}_{o, o}$ for compact operators if and only if $X^{*}$ is Fréchet differentiable. Let us notice that $X^{*}$ is Fréchet differentiable if and only if $X$ is strictly convex and $(X, \mathbb{K})$ has the $\mathbf{L}_{o, o}$ (see $[9$, Theorem 2.5]). Therefore, Theorem A generalizes [17, Theorem 2.3] as we no longer need strict convexity on $X$. On the other hand, [16, Theorem 2.12] says that if $X$ is a reflexive space which satisfies the Kadec-Klee property, then $(X, Y)$ has the $\mathbf{L}_{o, o}$ for compact operators for every $Y$. This is also covered by our Theorem A since whenever $X$ is a reflexive space satisfying the Kadec-Klee property, the pair $(X, \mathbb{K})$ satisfies the $\mathbf{L}_{o, o}$ (see [9, Propositions 2.2 and 2.6]).

We now present the proof of Theorem B.

Proof of Theorem B. (a) $\Rightarrow$ (b). If we assume (a), then we have that $X$ and $Y$ are both reflexive, and that every operator from $X$ into $Y^{*}$ is compact. So, $\mathcal{L}\left(X, Y^{*}\right)=\left(X \widehat{\otimes}_{\pi} Y\right)^{*}$ is reflexive by Ryan [15, Theorem 4.19]. Then, $X \widehat{\otimes}_{\pi} Y$ is reflexive.

(b) $\Rightarrow$ (a). If $X \widehat{\otimes}_{\pi} Y$ is reflexive, then so is $\mathcal{L}\left(X, Y^{*}\right)=\left(X \widehat{\otimes}_{\pi} Y\right)^{*}$. Since $\left(X, Y^{*}\right)$ has the pointwise-BCAP (or either $X$ or $Y$ has the AP), we have that $\mathcal{L}\left(X, Y^{*}\right)=\mathcal{K}\left(X, Y^{*}\right)$ (see [10, the diagram on pg.4] for the pointwise-BCAP assumption and [15, Theorem 4.21] for the AP assumption).

(a) $\Rightarrow$ (c). Suppose that $\mathcal{L}\left(X, Y^{*}\right)=\mathcal{K}\left(X, Y^{*}\right)$ and assume that both $(X, \mathbb{K})$ and $(Y, \mathbb{K})$ satisfy the $\mathbf{L}_{o, o}$ for linear functionals. By contradiction, let us assume that $(X \times Y ; \mathbb{K})$ fails to have the $\mathbf{L}_{o, o}$ for bilinear forms. Then, there exist $\varepsilon_{0}>0, B \in \mathcal{B}(X \times Y ; \mathbb{K})$ with $\|B\|=1$, and $\left(x_{n}, y_{n}\right)_{n=1}^{\infty} \subseteq S_{X} \times S_{Y}$ such that

$$
1 \geq B\left(x_{n}, y_{n}\right) \geq 1-\frac{1}{n}
$$

and whenever $(u, v) \subset S_{X} \times S_{Y}$ is such that $B(u, v)=1$, we have that

$$
\left\|u-x_{n}\right\| \geq \varepsilon_{0} \quad \text { or } \quad\left\|v-y_{n}\right\| \geq \varepsilon_{0} .
$$

Since $X$ and $Y$ are reflexive and both $\left(x_{n}\right)_{n=1}^{\infty}$ and $\left(y_{n}\right)_{n=1}^{\infty}$ are bounded, we may assume (and we do) that $x_{n} \stackrel{w}{\longrightarrow} x_{0}$ and $y_{n} \stackrel{w}{\longrightarrow} y_{0}$ for some $x_{0} \in B_{X}$ and $y_{0} \in B_{Y}$. Let $T \in \mathcal{L}\left(X, Y^{*}\right)=\left(X \widehat{\otimes}_{\pi} Y\right)^{*}$ be arbitrary. By assumption, we have that $T \in \mathcal{K}\left(X, Y^{*}\right)$. Since $x_{n} \stackrel{w}{\longrightarrow} x_{0}$ and $T$ is compact, we have that $T\left(x_{n}\right) \stackrel{\|\cdot\|}{\longrightarrow} T\left(x_{0}\right)$ and then since

$$
\left|T\left(x_{n}\right)\left(y_{n}\right)-T\left(x_{0}\right)\left(y_{0}\right)\right| \leq\left\|T\left(x_{n}\right)-T\left(x_{0}\right)\right\|\left\|y_{n}\right\|+\left|T\left(x_{0}\right)\left(y_{n}\right)-T\left(x_{0}\right)\left(y_{0}\right)\right|,
$$


we have that

$$
T\left(x_{n}\right)\left(y_{n}\right) \longrightarrow T\left(x_{0}\right)\left(y_{0}\right)
$$

as $n \rightarrow \infty$ for every $T \in\left(X \widehat{\otimes}_{\pi} Y\right)^{*}=\mathcal{L}\left(X, Y^{*}\right)=\mathcal{K}\left(X, Y^{*}\right)$. This means that $x_{n} \otimes y_{n} \stackrel{w}{\longrightarrow} x_{0} \otimes y_{0}$. In particular, since $B \in \mathcal{B}(X \times Y ; \mathbb{R})=\left(X \widehat{\otimes}_{\pi} Y\right)^{*}$, we have that $B\left(x_{n}, y_{n}\right) \longrightarrow B\left(x_{0}, y_{0}\right)$ and by $(2.2), B\left(x_{0}, y_{0}\right)=1$. In particular, $x_{0} \in S_{X}$ and $y_{0} \in S_{Y}$.

Let us consider $T_{B} \in \mathcal{L}\left(X, Y^{*}\right)$ and $S_{B} \in \mathcal{L}\left(Y, X^{*}\right)$ to be the associated linear operators to the bilinear form $B$. We have that

$$
T_{B}^{*}\left(y_{0}\right)\left(x_{0}\right)=T_{B}\left(x_{0}\right)\left(y_{0}\right)=B\left(x_{0}, y_{0}\right)=1,
$$

which shows that $T_{B}^{*}\left(y_{0}\right) \in S_{X^{*}}$. Analogously, $S_{B}^{*}\left(x_{0}\right)\left(y_{0}\right)=1$ and $S_{B}^{*}\left(x_{0}\right) \in$ $S_{Y^{*}}$.

Claim: We have that

$(\star) T_{B}^{*}\left(y_{0}\right)\left(x_{n}\right) \longrightarrow 1$ as $n \rightarrow \infty$.

$(\star \star) S_{B}^{*}\left(x_{0}\right)\left(y_{n}\right) \longrightarrow 1$ as $n \rightarrow \infty$.

We only prove $(\star)$ since $(\star \star)$ is analogous. As $T_{B}^{*}$ is a compact operator and $y_{n} \stackrel{w}{\longrightarrow} y_{0}$, we have that $T_{B}^{*}\left(y_{n}\right) \stackrel{\|\cdot\|}{\longrightarrow} T_{B}^{*}\left(y_{0}\right)$. At the same time, by $(2.4)$ we have that

$$
T_{B}^{*}\left(y_{n}\right)\left(x_{n}\right)=T_{B}\left(x_{n}\right)\left(y_{n}\right) \longrightarrow T_{B}\left(x_{0}\right)\left(y_{0}\right)=1
$$

as $n \rightarrow \infty$. Therefore,

$$
\begin{aligned}
\left|T_{B}^{*}\left(y_{n}\right)\left(x_{n}\right)-T_{B}^{*}\left(y_{0}\right)\left(x_{n}\right)\right| & =\left|\left(T_{B}^{*}\left(y_{n}\right)-T_{B}^{*}\left(y_{0}\right)\left(x_{n}\right)\right)\right| \\
& \leq\left\|T_{B}^{*}\left(y_{n}\right)-T_{B}^{*}\left(y_{0}\right)\right\| \longrightarrow 0
\end{aligned}
$$

and so $T_{B}^{*}\left(y_{0}\right)\left(x_{n}\right) \longrightarrow 1$ as $n \rightarrow \infty$.

Let us prove that $\left\|x_{n}-x\right\| \longrightarrow \infty$ as $n \rightarrow \infty$. Assume first that $X$ satisfies the Kadec-Klee property. Since $x_{0} \in S_{X}$ and $x_{n} \stackrel{w}{\longrightarrow} x_{0}$, we have that $\left\|x_{n}-x_{0}\right\| \rightarrow 0$ as $n \rightarrow \infty$. We prove that the same holds if $X$ is taken to be strictly convex. Indeed, by using $(\star)$, we have that $T_{B}^{*}\left(y_{0}\right)\left(x_{n}\right) \longrightarrow 1$ as $n \rightarrow \infty$. Since $(X, \mathbb{K})$ satisfies the $\mathbf{L}_{o, o}$ and $X$ is strictly convex, we have that $X^{*}$ is Fréchet differentiable (see [9, Theorem 2.5.(b)] and then, by the Šmulyan lemma, we have that $\left\|x_{n}-x_{0}\right\| \longrightarrow 0$ as $n \rightarrow \infty$ as desired.

To conclude the proof of this implication, let us set $y_{0}^{*}:=S_{B}^{*}\left(x_{0}\right) \in S_{Y^{*}}$. Then, $y_{0}^{*}\left(y_{0}\right)=1$ and $y_{0}^{*}\left(y_{n}\right) \longrightarrow 1$ as $n \rightarrow \infty$ by $(\star \star)$. Since $(Y, \mathbb{K})$ has the $\mathbf{L}_{o, o}$ for linear functionals, there is $\left(y_{n}^{\prime}\right) \subseteq S_{Y}$ such that $y_{0}^{*}\left(y_{n}^{\prime}\right)=1$ and $\left\|y_{n}^{\prime}-y_{n}\right\| \longrightarrow 0$ as $n \rightarrow \infty$. This means that

$$
1=y_{0}^{*}\left(y_{n}^{\prime}\right)=S_{B}^{*}\left(x_{0}\right)\left(y_{n}^{\prime}\right)=B\left(x_{0}, y_{n}^{\prime}\right) .
$$

Since $\left\|x_{n}-x_{0}\right\| \longrightarrow 0$ and $\left\|y_{n}^{\prime}-y_{n}\right\| \longrightarrow 0$ as $n \rightarrow \infty$, we get a contradiction with (2.3).

$(\mathrm{c}) \Rightarrow(\mathrm{d})$. Suppose that $(X \times Y ; \mathbb{K})$ has the $\mathbf{L}_{o, o}$ for bilinear forms. To prove that $\left(X \widehat{\otimes}_{\pi} Y, \mathbb{K}\right)$ has the $\mathbf{L}_{o, o}$ for linear functionals, let us fix $B \in\left(X \widehat{\otimes}_{\pi} Y\right)^{*}=$ 
$\mathcal{B}(X \times Y ; \mathbb{K})$ with $\|B\|=1$. By hypothesis, given $\varepsilon \in(0,1)$, there exists $\eta(\varepsilon, B)>0$. We use similar arguments from [6, Proposition 4.3].

Let $z \in S_{X \widehat{\otimes}_{\pi} Y}$ be such that

$$
\operatorname{Re}\langle B, z\rangle>1-\frac{\eta(\varepsilon, B)^{2}}{2} .
$$

By Ryan [15, Proposition 2.8] we can find $\left(x_{n}\right)_{n=1}^{\infty} \subseteq S_{X},\left(y_{n}\right)_{n=1}^{\infty} \subseteq S_{Y}$, and $\left(\lambda_{n}\right)_{n=1}^{\infty} \subseteq \mathbb{R}^{+}$such that $z=\sum_{n=1}^{\infty} \lambda_{n} x_{n} \otimes y_{n}$ and such that

$$
\sum_{n=1}^{\infty} \lambda_{n}<1+\frac{\eta(\varepsilon, B)^{2}}{2}
$$

Consider the sets

$$
I:=\left\{n \in \mathbb{N}: \operatorname{Re} B\left(x_{n}, y_{n}\right)>1-\eta(\varepsilon, B)\right\}
$$

and $J:=I^{c}$. Hence, we have that

$$
\begin{aligned}
1-\frac{\eta(\varepsilon, B)^{2}}{2}<\operatorname{Re}\langle B, z\rangle & =\sum_{n=1}^{\infty} \lambda_{n} \operatorname{Re} B\left(x_{n}, y_{n}\right) \\
& =\sum_{n \in I} \lambda_{n} \operatorname{Re} B\left(x_{n}, y_{n}\right)+\sum_{n \in J} \lambda_{n} \operatorname{Re} B\left(x_{n}, y_{n}\right) \\
& \leq \sum_{n \in I} \lambda_{n}+(1-\eta(\varepsilon, B)) \sum_{n \in J} \lambda_{n} \\
& =\sum_{n=1}^{\infty} \lambda_{n}-\eta(\varepsilon, B) \sum_{n \in J} \lambda_{n} \\
& <1+\frac{\eta(\varepsilon, B)^{2}}{2}-\eta(\varepsilon, B) \sum_{n \in J} \lambda_{n},
\end{aligned}
$$

that is, $\eta(\varepsilon, B) \sum_{n \in J} \lambda_{n}<\eta(\varepsilon, B)^{2}$ and then

$$
\sum_{n \in J} \lambda_{n}<\eta(\varepsilon, B)
$$

Notice that for each $n \in I$, we have that $\operatorname{Re} B\left(x_{n}, y_{n}\right)>1-\eta(\varepsilon, B)$. Then, by hypothesis, there exists $\left(x_{n}^{\prime}, y_{n}^{\prime}\right)_{n \in I} \subseteq S_{X} \times S_{Y}$ such that

$$
\left|B\left(x_{n}^{\prime}, y_{n}^{\prime}\right)\right|=1, \quad\left\|x_{n}^{\prime}-x_{n}\right\|<\varepsilon, \quad \text { and } \quad\left\|y_{n}^{\prime}-y_{n}\right\|<\varepsilon .
$$


Let us write $B\left(x_{n}^{\prime}, y_{n}^{\prime}\right)=e^{i \theta_{n}}$ with some $\theta_{n} \in \mathbb{R}$ for every $n \in I$. Let us notice that, for each $n \in I$, we have

$$
\begin{aligned}
1-\eta(\varepsilon, B)<\operatorname{Re} B\left(x_{n}, y_{n}\right) & =\operatorname{Re} B\left(x_{n}-x_{n}^{\prime}+x_{n}^{\prime}, y_{n}\right) \\
& =\operatorname{Re} B\left(x_{n}-x_{n}^{\prime}, y_{n}\right)+\operatorname{Re} B\left(x_{n}^{\prime}, y_{n}\right) \\
& \leq\left\|x_{n}-x_{n}^{\prime}\right\|+\operatorname{Re} B\left(x_{n}^{\prime}, y_{n}-y_{n}^{\prime}+y_{n}^{\prime}\right) \\
& =\left\|x_{n}-x_{n}\right\|^{\prime}+\operatorname{Re} B\left(x_{n}^{\prime}, y_{n}-y_{n}^{\prime}\right)+\operatorname{Re} B\left(x_{n}^{\prime}, y_{n}^{\prime}\right) \\
& \leq\left\|x_{n}-x_{n}^{\prime}\right\|+\left\|y_{n}-y_{n}^{\prime}\right\|+\operatorname{Re} B\left(x_{n}^{\prime}, y_{n}^{\prime}\right) \\
& <2 \varepsilon+\operatorname{Re} B\left(x_{n}^{\prime}, y_{n}^{\prime}\right)
\end{aligned}
$$

that is, $1-\operatorname{Re} B\left(x_{n}^{\prime}, y_{n}^{\prime}\right)<2 \varepsilon+\eta(\varepsilon, B)$ for each $n \in I$. Now, since $1=$ $\left|B\left(x_{n}^{\prime}, y_{n}^{\prime}\right)\right|^{2}=\operatorname{Re} B\left(x_{n}^{\prime}, y_{n}^{\prime}\right)^{2}+\operatorname{Im} B\left(x_{n}^{\prime}, y_{n}^{\prime}\right)^{2}$, we have that, for every $n \in I$,

$$
1>1-2 \varepsilon-\eta(\varepsilon, B)+\operatorname{Im} B\left(x_{n}^{\prime}, y_{n}^{\prime}\right)^{2}
$$

which implies that $\operatorname{Im} B\left(x_{n}^{\prime}, y_{n}^{\prime}\right)^{2}<2 \varepsilon+\eta(\varepsilon, B)$. Thus, if $n \in I$, we have that

$$
\begin{aligned}
\left|1-e^{i \theta}\right| & =\left|1-B\left(x_{n}^{\prime}, y_{n}^{\prime}\right)\right| \\
& =\sqrt{\left(1-\operatorname{Re} B\left(x_{n}^{\prime}, y_{n}^{\prime}\right)\right)^{2}+\operatorname{Im} B\left(x_{n}^{\prime}, y_{n}^{\prime}\right)^{2}} \\
& <\sqrt{(2 \varepsilon+\eta(\varepsilon, B))^{2}+2 \varepsilon+\eta(\varepsilon, B)} \\
& <\sqrt{4 \varepsilon+2 \eta(\varepsilon, B)} .
\end{aligned}
$$

Now, let us define

$$
z^{\prime}:=\sum_{n \in I} \lambda_{n} e^{-i \theta_{n}} x_{n}^{\prime} \otimes y_{n}^{\prime} \in X \widehat{\otimes}_{\pi} Y .
$$

We have that

$$
\left\langle B, z^{\prime}\right\rangle=\sum_{n \in I} \lambda_{n} e^{-i \theta_{n}} B\left(x_{n}^{\prime}, y_{n}^{\prime}\right)=\sum_{n \in I} \lambda_{n}=\left\|z^{\prime}\right\|_{\pi}
$$

On the other hand, since

$$
\begin{aligned}
\left\|x_{n}^{\prime} \otimes y_{n}^{\prime}-x_{n} \otimes y_{n}\right\| & \leq\left\|x_{n}^{\prime} \otimes y_{n}^{\prime}-x_{n}^{\prime} \otimes y_{n}\right\|+\left\|x_{n}^{\prime} \otimes y_{n}-x_{n} \otimes y_{n}\right\| \\
& \leq\left\|y_{n}^{\prime}-y_{n}\right\|+\left\|x_{n}^{\prime}-x_{n}\right\| \\
& <2 \varepsilon
\end{aligned}
$$


we have that (here we use $(2.5)$ and the fact that $\left|1-e^{i \theta}\right|<\sqrt{4 \varepsilon+2 \eta(\varepsilon, B)}$ )

$$
\begin{aligned}
\left\|z^{\prime}-z\right\|_{\pi} & =\left\|\sum_{n \in I} \lambda_{n} e^{-i \theta_{n}} x_{n}^{\prime} \otimes y_{n}^{\prime}-\sum_{n \in I} \lambda_{n} x_{n} \otimes y_{n}-\sum_{n \in J} \lambda_{n} x_{n} \otimes y_{n}\right\| \\
& \leq\left\|\sum_{n \in I} \lambda_{n}\left(e^{-i \theta_{n}} x_{n}^{\prime} \otimes y_{n}^{\prime}-x_{n} \otimes y_{n}\right)\right\|+\sum_{n \in J} \lambda_{n} \\
& \leq \sum_{n \in I} \lambda_{n}\left|e^{-i \theta_{n}}-1\right|+\left\|\sum_{n \in I} \lambda_{n}\left(x_{n}^{\prime} \otimes y_{n}^{\prime}-x_{n} \otimes y_{n}\right)\right\|+\sum_{n \in J} \lambda_{n} \\
& <(\sqrt{4 \varepsilon+2 \eta(\varepsilon, B)}) \sum_{n \in I} \lambda_{n}+2 \varepsilon \sum_{n \in I} \lambda_{n}+\sum_{n \in J} \lambda_{n} \\
& <(\sqrt{4 \varepsilon+2 \eta(\varepsilon, B)})\left(1+\frac{\eta(\varepsilon, B)^{2}}{2}\right)+2 \varepsilon\left(1+\frac{\eta(\varepsilon, B)^{2}}{2}\right)+\eta(\varepsilon, B) \\
& =(\sqrt{4 \varepsilon+2 \eta(\varepsilon, B)}+2 \varepsilon)\left(1+\frac{\eta(\varepsilon, B)^{2}}{2}\right)+\eta(\varepsilon, B) .
\end{aligned}
$$

In particular, we have that $\left\|z^{\prime}\right\|>0$ and we may define $z^{\prime \prime}:=\frac{z^{\prime}}{\left\|z^{\prime}\right\|} \in S_{X \widehat{\otimes}_{\pi} Y}$. Notice that, since

$$
\left\|z^{\prime \prime}-z^{\prime}\right\|=\left\|\frac{z^{\prime}}{\left\|z^{\prime}\right\|}-z^{\prime}\right\|=\left|1-\left\|z^{\prime}\right\|\right| \leq\left\|z-z^{\prime}\right\|
$$

we have that

$$
\begin{aligned}
\left\|z^{\prime \prime}-z\right\| & \leq\left\|z^{\prime \prime}-z^{\prime}\right\|+\left\|z^{\prime}-z\right\| \\
& <2\left\|z^{\prime}-z\right\| \\
& <2(\sqrt{4 \varepsilon+2 \eta(\varepsilon, B)}+2 \varepsilon)\left(1+\frac{\eta(\varepsilon, B)^{2}}{2}\right)+2 \eta(\varepsilon, B) .
\end{aligned}
$$

Finally, notice that

$$
\left\langle B, z^{\prime \prime}\right\rangle=\left\langle B, \frac{z^{\prime}}{\left\|z^{\prime}\right\|}\right\rangle=1 .
$$

This shows that $\left(X \widehat{\otimes}_{\pi} Y, \mathbb{R}\right)$ satisfies the $\mathbf{L}_{o, o}$ for linear functionals.

$(\mathrm{d}) \Rightarrow(\mathrm{b})$. Suppose that $\left(X \widehat{\otimes}_{\pi} Y, \mathbb{K}\right)$ has the $\mathbf{L}_{o, o}$ for linear functionals. By Dantas et al. [9, Theorem 2.3], $X \widehat{\otimes}_{\pi} Y$ is reflexive and $\left(X \widehat{\otimes}_{\pi} Y\right)^{*}$ is SSD. Since $X^{*}, Y^{*}$ are closed subspaces of $\left(X \widehat{\otimes}_{\pi} Y\right)^{*}=\mathcal{L}\left(X, Y^{*}\right)=\mathcal{L}\left(Y, X^{*}\right)$, we have that both $X^{*}, Y^{*}$ are $\operatorname{SSD}[11]$. Therefore, both $(X, \mathbb{K})$ and $(Y, \mathbb{K})$ satisfy the $\mathbf{L}_{o, o}$ for linear functionals.

Remark 2.2. In Theorem B, the assumptions that $X$ or $Y$ has the AP or that the pair $\left(X, Y^{*}\right)$ satisfies the pointwise-BCAP are only used to get that $(\mathrm{b}) \Rightarrow$ (a); on the other hand, implications (a) $\Rightarrow(\mathrm{c}) \Rightarrow(\mathrm{d}) \Rightarrow(\mathrm{b})$ are valid without these assumptions (notice that $(\mathrm{c}) \Rightarrow(\mathrm{d}) \Rightarrow(\mathrm{b})$ are valid for general Banach spaces while in $(\mathrm{a}) \Rightarrow(\mathrm{c})$ we assume that $X$ is strictly convex or that satisfies the Kadec-Klee property). 
Remark 2.3. The approximation properties in Theorem B are technical assumptions we need in order to guarantee that the equality $\mathcal{L}\left(X, Y^{*}\right)=$ $\mathcal{K}\left(X, Y^{*}\right)$ holds true. In fact, this is what we use to get $(\mathrm{b}) \Rightarrow$ (a). Let us notice also that, as far as we know, it is an open problem whether the fact $X \widehat{\otimes}_{\pi} Y$ is reflexive implies that $\mathcal{L}\left(X, Y^{*}\right)=\mathcal{K}\left(X, Y^{*}\right)$ is true for general reflexive spaces $X$ and $Y$. A positive answer for this problem would provide us a general characterization for Theorem B.

As an immediate consequence of Theorem B, we have the following corollary. Notice that item (a) below was proved also in [8, Theorem 2.7.(b)].

Corollary 2.1. Let $1<p, q<\infty$ and let $q^{\prime}$ be the conjugate index of $q$.

(a) $\left(\ell_{p} \times \ell_{q} ; \mathbb{K}\right)$ satisfies the $\mathbf{L}_{o, o}$ for bilinear forms if and only if $p>q^{\prime}$.

(b) $\left(L_{p}(\mu), L_{q}(\nu) ; \mathbb{K}\right)$ fails the $\mathbf{L}_{o, o}$ for bilinear forms for not purely atomic measures $\mu, \nu$.

Proof. Under the assumption of (a), we have that the projective tensor product $\ell_{p} \widehat{\otimes}_{\pi} \ell_{q}$ is reflexive (see [15, Corollary 4.24]). For (b), since $L_{p}(\mu) \widehat{\otimes}_{\pi} L_{q}(\nu)$ contains complemented isomorphic copies of $\ell_{1}$ for every $p, q$, it is never reflexive (see [15, Theorem 4.21 and Corollary 4.22]). Therefore, both items follow immediately by applying Theorem B.

Let us conclude the paper by commenting on the $\mathbf{L}_{o, o}$ for different classes of functions. Let $X$ and $Y$ be Banach spaces. In $\mathcal{B}(X \times Y ; \mathbb{K})$, as we have seen in Theorem B, one can consider:

(A) the $\mathbf{L}_{o, o}$ for linear functionals seeing $\mathcal{B}(X \times Y ; \mathbb{K})$ as $\left(X \widehat{\otimes}_{\pi} Y\right)^{*}$,

(B) the $\mathbf{L}_{o, o}$ for operators seeing $\mathcal{B}(X \times Y ; \mathbb{K})$ as $\mathcal{L}\left(X, Y^{*}\right)$, and, of course,

(C) the $\mathbf{L}_{o, o}$ for bilinear forms.

We have the following relation between properties $(\mathrm{A}),(\mathrm{B})$, and $(\mathrm{C})$ :

- General implications. Clearly, we have that $(\mathrm{C}) \Rightarrow(\mathrm{B})$ by considering the associated bilinear for $B_{T} \in \mathcal{B}(X \times Y ; \mathbb{K})$ of a given operator $T \in$ $\mathcal{L}\left(X, Y^{*}\right)$. Also, by our Theorem B (implication $\left.(\mathrm{c}) \Rightarrow(\mathrm{d})\right)$ and noticing that, for this implication, we do not need any assumption on $X$ besides reflexivity (not even approximation property assumptions), we also have that $(\mathrm{C}) \Rightarrow(\mathrm{A})$.

- Not true implications. (B) does not imply (A) or (C) in general. Indeed, by [1, Theorem 2.4.10], for every $1<p<\infty$, we have that $\mathcal{L}\left(c_{0}, \ell_{p}\right)=$ $\mathcal{K}\left(c_{0}, \ell_{p}\right)=\mathcal{L}\left(\ell_{p^{\prime}}, \ell_{1}\right)$, where $p^{\prime}$ is the conjugate index of $p$. We have that $\left(\ell_{p^{\prime}}, \ell_{1}\right)$ has the $\mathbf{L}_{o, o}$ for operators by Theorem A (since $\left(\ell_{p^{\prime}}, \mathbb{K}\right)$ has the $\mathbf{L}_{o, o}$ for linear functionals) but neither $\left(\ell_{p^{\prime}} \times c_{0} ; \mathbb{K}\right)$ nor $\left(\ell_{p^{\prime}} \widehat{\otimes}_{\pi} c_{0} ; \mathbb{K}\right)$ can have the $\mathbf{L}_{o, o}$ for bilinear forms and for linear functionals, respectively, since $c_{0}$ is not reflexive.

- Implications with extra assumptions. Assume that either $X$ or $Y$ has the AP, or that the pair $\left(X, Y^{*}\right)$ has the pointwise-BCAP. In this case, implication $(\mathrm{A}) \Rightarrow(\mathrm{B})$ holds. Indeed, if $\left(X \widehat{\otimes}_{\pi} Y ; \mathbb{K}\right)$ has the $\mathbf{L}_{o, o}$ for linear 
functionals, then $X \widehat{\otimes}_{\pi} Y$ must be reflexive and, by one of our assumptions, every operator from $X$ into $Y^{*}$ is compact and by Theorem B, the pair $(X, \mathbb{K})$ has the $\mathbf{L}_{o, o}$ for linear functionals. By Theorem A, the pair $\left(X, Y^{*}\right)$ has the $\mathbf{L}_{o, o}$ for operators. Finally, if $X$ or $Y$ has the $\mathrm{AP}$ (or $\left(X, Y^{*}\right)$ has the pointwise-BCAP) and $X$ or $Y$ is stricly convex, then $(\mathrm{A}) \Rightarrow(\mathrm{C})$.

\section{Acknowledgements}

The authors are thankful to Mingu Jung and Martin Mazzitelli for fruitful conversations on the topic of the paper. Also, they are deeply grateful to the anonymous referee who careful read the paper and suggested several improvements on Theorem B.

Funding Open Access funding provided thanks to the CRUE-CSIC agreement with Springer Nature. S. Dantas was supported by Spanish AEI Project PID2019-106529GB-I00 / AEI / 10.13039/501100011033 and also by PGC2018-093794-B-I00 (MCIU/AEI/FEDER, UE). A. Rueda Zoca was supported by Juan de la Cierva-Formación fellowship FJC2019-039973, by MTM2017-86182-P (Government of Spain, AEI/ FEDER, EU), by MICINN (Spain) Grant PGC2018-093794-B-I00 (MCIU, AEI, FEDER, UE), by Fundación Séneca, ACyT Región de Murcia grant 20797/PI/18, by Junta de Andalucía Grant A-FQM-484-UGR18 and by Junta de Andalucía Grant FQM0185 .

\section{Declaration}

Conflict of interests All authors declare that they have no conflict of interest.

Open Access. This article is licensed under a Creative Commons Attribution 4.0 International License, which permits use, sharing, adaptation, distribution and reproduction in any medium or format, as long as you give appropriate credit to the original author(s) and the source, provide a link to the Creative Commons licence, and indicate if changes were made. The images or other third party material in this article are included in the article's Creative Commons licence, unless indicated otherwise in a credit line to the material. If material is not included in the article's Creative Commons licence and your intended use is not permitted by statutory regulation or exceeds the permitted use, you will need to obtain permission directly from the copyright holder. To view a copy of this licence, visit http://creativecommons.org/ licenses/by $/ 4.0 /$.

\section{References}

[1] Albiac, F., Kalton, N.: Topics in Banach Space Theory. Graduate Texts in Mathematics 233, Springer, New York (2006) 
[2] Bishop, E., Phelps, R.R.: A proof that every Banach space is subreflexive. Bull. Am. Math. Soc. 67, 97-98 (1961)

[3] Bollobás, B.: An extension to the theorem of Bishop and Phelps. Bull. Lond. Math. Soc. 2, 181-182 (1970)

[4] Dantas, S.: Some kind of Bishop-Phelps-Bollobás property. Math. Nachr. 290(56), 774-784 (2017)

[5] Dantas, S., García-Lirola, L.C., Jung, M., Rueda-Zoca, A.: On norm-attainment in (symmetric) tensor products. Preprint available on arXiv, arXiv:2104.06841

[6] Dantas, S., Jung, M., Roldán, Ó., Rueda-Zoca, A.: Norm-attaining tensors and nuclear operators, to be formally accepted in Mediterranean Journal of Mathematics. Preprint available on arXiv arXiv:2006.09871

[7] Dantas, S., Kadets, V., Kim, S.K., Lee, H.J., Martín, M.: There is no operator wise version of the Bishop-Phelps-Bollobás property. Linear Multilinear Algebra 68(9), 1767-1778 (2020)

[8] Dantas, S., Kim, S.K., Lee, H.J., Mazzitelli, M.: Strong subdifferentiability and local Bishop-Phelps-Bollobás properties. Rev. R. Acad. Cienc. Exactas Fís. Nat. Ser. A Mat. RACSAM 114(2) (2020). Paper No. 47, 16 pp

[9] Dantas, S., Kim, S.K., Lee, H.J., Mazzitelli, M.: Local Bishop-Phelps-Bollobás properties. J. Math. Anal. Appl. 468, 304-323 (2018)

[10] Dantas, S., Jung, M., Martínez-Cervantes, G.: On the existence of non-normattaining operators, Journal of the Institute of Mathematics of Jussieu, 1-13. https://doi.org/10.1017/S1474748021000311

[11] Franchetti, C., Payá, R.: Banach spaces with strongly subdifferentiable norm. Boll. Un. Mat. Ital. VII-B, 45-70 (1993)

[12] Godefroy, G., Montesinos, V., Zizler, V.: Strong subdifferentiability of norms and geometry of Banach spaces. Comment. Math. Univ. Carolin. 36(3), 493-502 (1995)

[13] Kim, S.K., Lee, H.J.: Uniform convexity and the Bishop-Phelps-Bollobás property. Can. J. Math. 66, 373-386 (2014)

[14] Martín, M.: Norm-attaining compact operators. J. Funct. Anal. 267, 1585-1592 (2014)

[15] Ryan, R.A.: Introduction to Tensor Products of Banach Spaces. Springer Monographs in Mathematics, Springer, London (2002)

[16] Sain, D.: Smooth points in operator spaces and some Bishop-Phelps-Bollobás type theorems in Banach spaces. Oper. Matrices 13(2), 433-445 (2019)

[17] Talponen, J.: Note on a kind of Bishop-Phelps-Bollobás property for operators. Preprint arXiv:1707.03251 


\section{Sheldon Dantas}

Departament de Matemàtiques, Institut Universitari de Matemàtiques i Aplicacions de Castelló (IMAC)

Universitat Jaume I

Campus del Riu Sec. s/n

12071 Castelló

Spain

e-mail: dantas@uji.es

Abraham Rueda Zoca

Departamento de Matemáticas

Universidad de Murcia

Campus de Espinardo

30100 Murcia

Spain

e-mail: abraham.rueda@um.es

URL: https://arzenglish. wordpress .com

Received: May 12, 2021.

Accepted: July 22, 2021.

Publisher's Note Springer Nature remains neutral with regard to jurisdictional claims in published maps and institutional affiliations. 